\title{
Geographical Variation of the Quality of the School Offer in the City of Mbujimayi (Dr Congo): Data Processing by the Method of Multivariate Spatial Analysis
}

\author{
Kayembe Mpinguyabo ${ }^{1}$, Kakese Kunyima ${ }^{2} \&$ Kanda Nkula $^{2}$ \\ ${ }^{1}$ Senior Lecturer at ISP / Mbujimayi and PhD candidate at the Faculty of Sciences, UNIKIN \\ ${ }^{2}$ Professors at the Faculty of Sciences, UNIKIN \\ Correspondence: Kayembe Mpinguyabo, ISP / Mbujimayi and $\mathrm{PhD}$ candidate at the Faculty of Sciences, \\ UNIKIN. E-mail: mpiguyabu@gmail.com
}

Received: October 23, 2016

Accepted: November 9, 2016

Online Published: February 28, 2017

doi:10.5539/jgg.v9n1p63

URL: http://doi.org/10.5539/jgg.v9n1p63

\begin{abstract}
This article presents spatial data related to the quality of school infrastructures in the city of Mbujimayi so as to bring out their characteristics and the typology of quarters which contain these infrastructures. The variables used are presented in the spatial information matrix. The principal components analysis and factorial analysis of correspondences helped make the description of associations based on these variables. The correlation matrix gave birth to channels of the strongest positive correlations $(r \geq 0.60)$ and resulted in the principal component analysis. The main results are:

- The cartography of quarters containing schools.

- The spatial disparity between the variables having degrees of affinity with the location and topography of adequate school site, sanitation, access to public services, and matching buildings. These variables are opposite to those related to unsanitary, localization and indecent topography of the site as well as non-school access to public services.

- A strong interaction between sanitation and access to public services, including running water and electricity, determining the quality of schools.
\end{abstract}

Keywords: Quality, educational offer, Mbujimayi

\section{Introduction}

Mbujimayi is a postcolonial city whose size is about $135.2 \mathrm{~km}^{2}$. Geographically located in the centre of the DR Congo, in the humid tropical zone (between $6^{\circ} 5^{\prime}$ and $6^{\circ} 10^{\prime}$ southward latitude and $23^{\circ} 27^{\prime}$ and $23^{\circ} 40$ eastward latitude), it is the capital city of the province of Kasai-Oriental. The city is peopled by about 3000000 inhabitants in agglomeration or some $4 \%$ of the total population of the DR Congo (Institut National des Statistiques). From age group perspective, in the first half of 2015, the population of Mbujimayi was made up of $58 \%$ of youth ( $0-18$ years old) of which $40 \%$ were schoolable (5-19 years old). In 2010 there were 303 school infrastructures comprising 693 schools of which 78 kingergartens, 428 primaries and 187 secondaries within the agglomeration ( see figure 1 below).

This study has its place in the domain of " area structuring of the education system in the city of Mbujimayi ". Although specific studies have been conducted on the educational system of the DR Congo including the Eastern Kasai in general, the education system of Mbujimayi, however, has got some characteristics of its own.

This article is a contribution to the deepening of knowledge about geographical variation as far as the quality of school offer in the city of Mbujimayi is concerned. It also looks at the typology of quarters that contain schools. Basically, this work revolves around a methodological approach based on the collection and multivariate spatial analysis of data on the quality of school offers. 


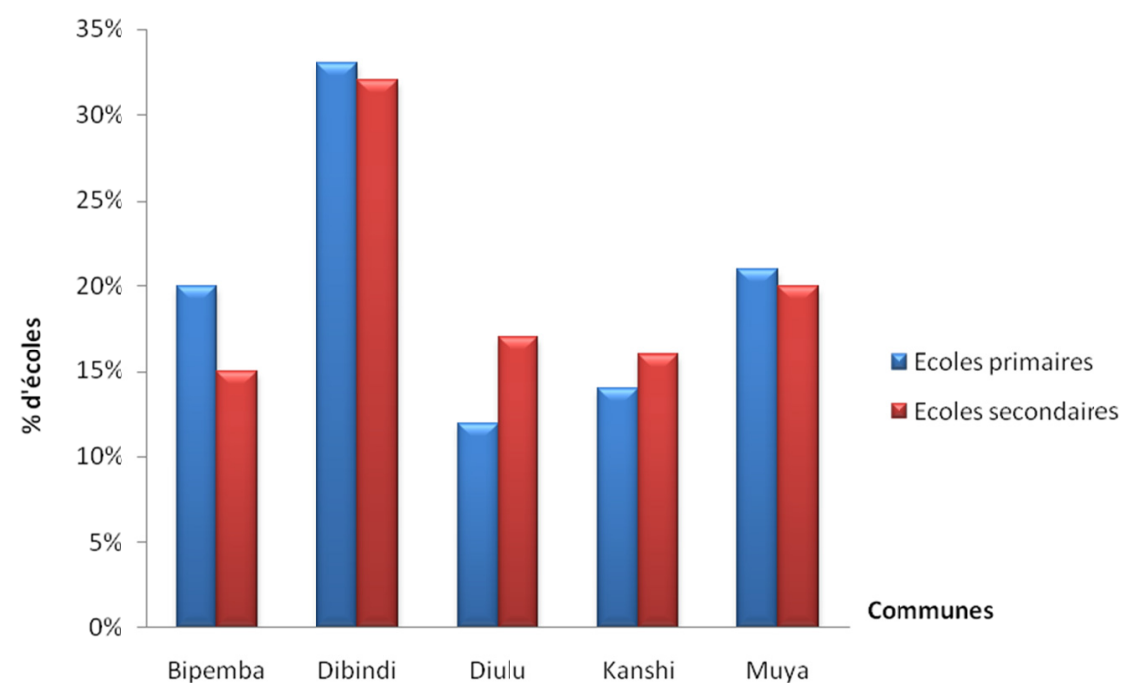

Figure 1. The city of Mbujimayi and its Schools in 2010

\section{Problematics of the Quality of School Offer}

From the outset, it is worth keeping in mind that the World Conference on Education was held in Jomtien from $5^{\text {th }}$ to $9^{\text {th }}$ March, 1990. In June 1996, Heads of African States and governments adopted the Harare Programme of Action for the Decade of Education in Africa (1997-2006). This programme revolved around the following priorities: - the equity and access to education; - the quality, relevance and effectiveness of education; additional modalities (alternative learning) and skills reinforcement. And from $26^{\text {th }}$ to $28^{\text {th }}$ April, 2000, the World Forum on Education was held in Dakar.

But the evaluations made on the Congolese Education System since 1990 have indicated that neither the objectives from Jomtien nor from Dakar have not been reached. Similarly the results of DR Congo are largely negative at the end of the "Decade of Education in Africa". All indicators of the effectiveness of the education system pointed out to the low performance (UNICEF, 2008). The country's economic situation has clearly had negative impacts on the development of the education system in general. This resulted in stagnation, or even dwindling of resources allocated to education, which resources are essential for the implementation of strategic objectives related to the development of the country's education sector.

According to Omasombo [(dir), 2014], the teaching in the province of Kasai Oriental is characterised by strong growth in the school population and a low density of school infrastructures per $\mathrm{km}^{2}$ which causes difficult access, less competent teachers, an acute shortage of teaching materials and the high cost of private education compared to per capita income and purchasing power in the bulk of its population.

Indeed, in the province of Kasai Oriental in general and in Mbujimayi city in particular, three fourth of the schools belong to the agreement sector. The state, meanwhile, is facing enormous difficulties in this sector and its school infrastructures are characterized by a very dilapidated state and the blatant insufficiency of equipment, to the extent that private initiatives intervene in this area rather to value funds to offset those deficiencies and inadequacies. Most private schools belong to traders and local NGOs.

The present study analyses the quality of school infrastructures. It focuses on the geographic variation of the school offer in Mbujimayi city with a goal of:

- identifying quarters with schools and

- sorting out the typology of these quarters.

\section{Research Methodology}

The present study analyzes the quality of school infrastructures which teaching types depend upon. "The different methods that allow the achievement of this goal can be grouped according to the nature of the variables that are now and then qualitative and quantitative. This means that any technical data representation presupposes the choice of a method for problems that can be described as an input - output system where the system $\mathrm{Y}$ output variables depend on $X$ input variables or more less controllable "( Ntumba, 2010). To do this, we used the descriptive and explanatory methods and quantitative methods. 


\subsection{The Descriptive and Explanatory Method}

This method is a participatory method in that it allows the shift from theory to practice. It consists in describing the facts as they are for the purpose of achieving the explanation by an inductive approach (Racine and Reymond, 1973). In other words, this method leads to the explanation of the variables under study thanks to many other explanatory variables. It also helps to explain several other independent variables from a numerical variable.

\subsection{Quantitative Methods}

These methods are concerned with the statistical analysis of data in order to compare the existing realities and those found on the field to see whether they match. Statistical analysis of quantified data has facilitated our task in the skimming, providing therefore the explanation in order to verify the empirical observations mathematically ( Kakese et al., 2012).

\subsection{The Analysis Methods}

Factorial analysis of multivariate data mainly covers two sets of techniques: the first set of techniques from the Euclidean geometry lead to the extraction of values and vectors of their own. The second set of techniques, so-called automatic classification are characterized by the choice of an index of proximity and an aggregation or disaggregation algorithm that achieve a score or classification tree as written in Pages et al. (1979).

Of these sets of techniques, the former occupies a special place because they are used either alone or together with the latter, while the latter are rarely applied alone (Escofier and Pages 1988). In this study, we are especially interested in the factorial analysis focusing on main component analysis (much used in English-speaking countries) and correspondence factorial analysis (popular in France), two most used methods.

According to Beguin (1979), factorial analysis is a method that seeks to achieve parsimony in the description while minimizing the loss of information. It constitutes, therefore, a help to the synthesis of data. The main components analysis and correspondence analysis are part of the basic methods of data analysis (Volle, 1978; Chadule Group, 1987; Paegelow,1996; Tenenhaus, 2007).

In essence, the goal of factorial analysis methods is to investigate subsets of small dimensions that best adjust the points of individuals and the clouds of points of variables.

This study draws on methods of factorial analysis of statistical data processed by statistics by using IT tools, capable of processing large masses of data on the characteristics of the investigation area.

Data analysis was mainly intended to present and handle multi-dimensional data of the studied variables. Thus, there may be three situations: absence of affinity, high binding or affinity and homogeneous groups.

\subsection{Geographic Information System (GIS)}

According to Burrough, "The G. I S. is a powerful instrumental equipment for the acquisition, representation, processing and transformation of localized data for the real world "(Paegelow, 1996).

The G. I. S. is "a high performance computer system for processing data and geographic information: capturing, storing, updating, deploying and manipulating information, and spatial data for making regional maps" (Rubenstein, 1996, Knox \& Marston, 2001). In short, it is storage of geographic information in the form of piled up folders (arranged in layers or sheets). So, they are information and digital data from several different sources on different subjects and at different scales, which can be in relationships and merged to the need for mapping and / or a reflection on area planning. This is possible because the G.S.I. has a great analytical capacity (Haring et al., 1992). Overall, the G.S.I. is a tool for storage, management and exploitation of spatial information; a computer software used in the production of maps from a spatial database.

In this study the following techniques were used:

- Library research, indispensible in any scientific work for accessing new knowledge made available by predecessors who have addressed similar topics,

- Direct observation consisted in inspecting the field in order to construct facts, events and phenomena to be studied;

- Data collection on the field was intended to establish a representative repository to better ground truth using a survey questionnaire;

- The direct collection georeferenced information was conducted mainly using a GPS "Garmin 60 CSX map " to capture simultaneously the geographic object and its location.

The spatial and cartographic data, from which this study is outcome were analyzed and treated at the laboratory 
METELSAT of Kinshasa by submitting them to 10.2 Arc GIS software.

\section{Presentation of Resultats}

The data presented in Table No. 1 below were collected on the field between 2008 and 2014. They are grouped in a double entry matrix of spatial or geographic information comprising quarters $\left(\mathrm{X}_{1} \ldots \mathrm{X}_{89}\right.$ and variables $\left(\mathrm{X}_{01} \ldots\right.$ $\mathrm{X}_{20}$ ) The map below (Figure No. 2) indicates the location of the different quarters containing school infrastructures.

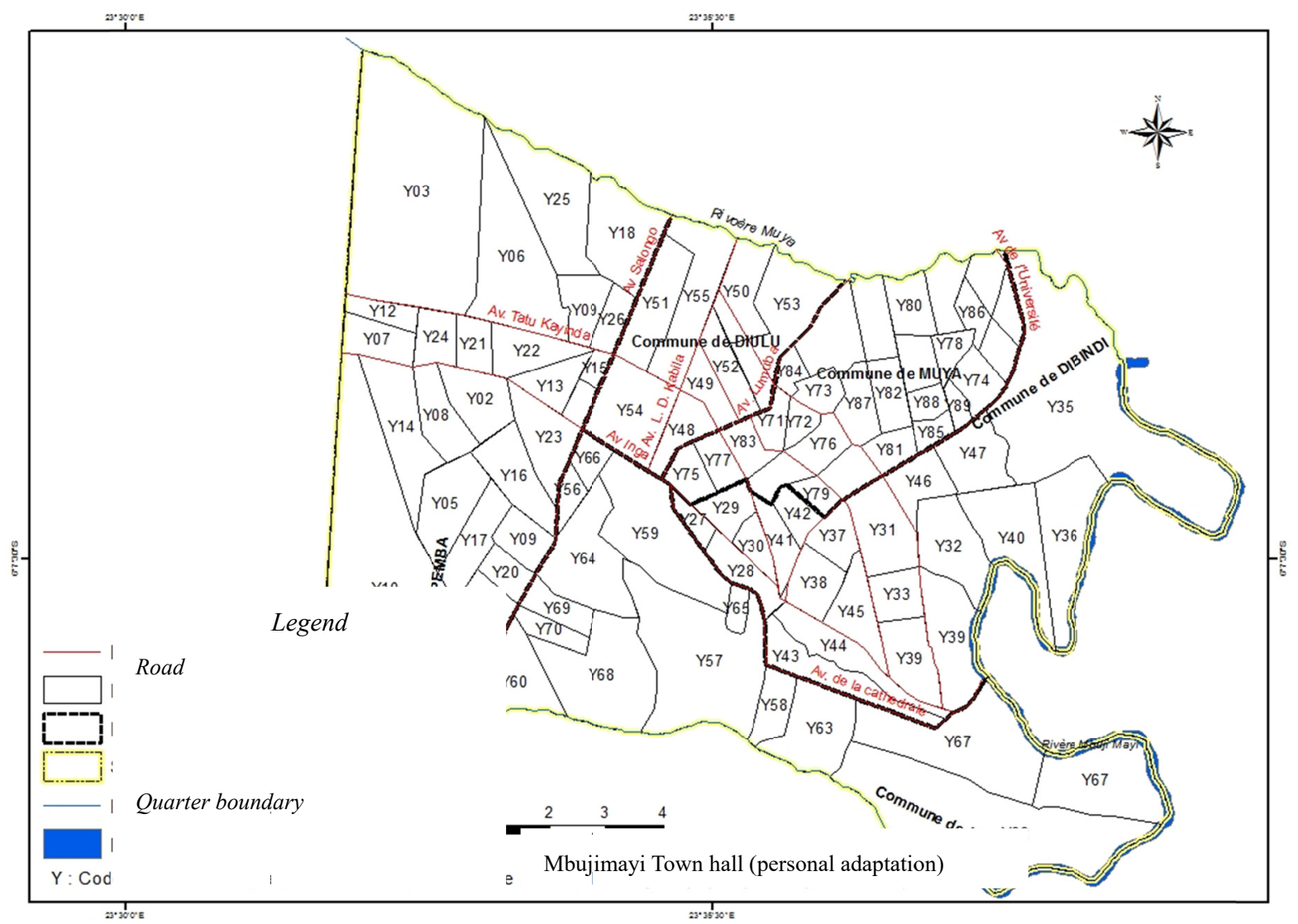

Figure 2. Mbujimayi (Politico-administrative map by the City Mayorship in 2010)

The quarters on the above map are:

$\mathrm{Y}_{01}$ : De la mission, $\mathrm{Y}_{02}$ : De la plaine, $\mathrm{Y}_{03}$ : Ditunga dietu, $\mathrm{Y}_{04}$ : Diovo, $\mathrm{Y}_{05}$ : Diwola, $\mathrm{Y}_{06}:$ Kabwatshia, $\mathrm{Y}_{07}$ : Kalundu, $\mathrm{Y}_{08}$ : Kanjiya, $\mathrm{Y}_{09}$ : Kankelenge, $\mathrm{Y}_{10}$ : Kanshi, $\mathrm{Y}_{11}$ : Kantu, $\mathrm{Y}_{12}$ : Lubanda, $\mathrm{Y}_{13}$ : Lubwebwe, $\mathrm{Y}_{14}$ : Makala, $\mathrm{Y}_{15}$ : Mpokolo, $\mathrm{Y}_{16}$ : Mulekayi, $\mathrm{Y}_{17}$ : Mulenda, $\mathrm{Y}_{18}$ : Muya, $\mathrm{Y}_{19}$ : Nzaba I, $\mathrm{Y}_{20}$ : Nzaba II, $\mathrm{Y}_{21}$ : Odia David, $Y_{22}$ : Regideso, $Y_{23}$ : RVA, $Y_{24}$ : Tshilela, $Y_{25}$ : Tshintunta, $Y_{26}$ : Tshiombela in Bipemba township. $Y_{27}$ : Bonzola I, $\mathrm{Y}_{28}$ : Bonzola II, $\mathrm{Y}_{29}$ : Dipumba I, $\mathrm{Y}_{30}$ : Dipumba II, $\mathrm{Y}_{31}$ : Kabongo I, $\mathrm{Y}_{32}$ : Kabongo II, $\mathrm{Y}_{33}$ : Kasavubu I, $\mathrm{Y}_{34}$ : Kasavubu II, $\mathrm{Y}_{35}$ : Lukelenge I, $\mathrm{Y}_{36}$ : Lukelenge II, $\mathrm{Y}_{37}$ : Mikela, $\mathrm{Y}_{38}$ : Minkoka, $\mathrm{Y}_{39}$ : Misesa I, $\mathrm{Y}_{40}$ : Misesa II, $\mathrm{Y}_{41}$ : Monzo I, $\mathrm{Y}_{42}$ : Monzo II, $\mathrm{Y}_{43}$ : Snel I, $\mathrm{Y}_{44}$ : Snel II, $\mathrm{Y}_{45}$ : Tshiya, $\mathrm{Y}_{46}$ : University I, $\mathrm{Y}_{47}$ : University II in the municipality of Dibindi. $\mathrm{Y}_{48}$ : Bubanji, $\mathrm{Y}_{49}$ : Dipa, $\mathrm{Y}_{50}$ : Hozana, $\mathrm{Y}_{51}$ : Lubuwa, $\mathrm{Y}_{52}$ : Lumumba, $\mathrm{Y}_{53}$ : Lusenga, $\mathrm{Y}_{54}$ : Masanka, $\mathrm{Y}_{55}$ : Nkulusa in Diulu township. $\mathrm{Y}_{56}$ : Bimpe, $\mathrm{Y}_{57}$ : Buzala, $\mathrm{Y}_{58}$ : Kasaï, $\mathrm{Y}_{59}$ : Kashala Bonzola, $\mathrm{Y}_{60}$ : Kananga, $\mathrm{Y}_{61}$ : Lubilanji I, $\mathrm{Y}_{62}$ : Lubilanji II, $\mathrm{Y}_{63}$ : Luzumu, $\mathrm{Y}_{64}$ : Makasi I, $\mathrm{Y}_{65}$ : Makasi II, $\mathrm{Y}_{66}$ : Mudiba, $\mathrm{Y}_{67}$ : Nyongolo, $\mathrm{Y}_{68}$ : Tshikisha, $\mathrm{Y}_{69}$ : Tubondo I, $\mathrm{Y}_{70}$ : Tubondo II in Kanshi township. $\mathrm{Y}_{71}$ : Badibanga Lukuni, $\mathrm{Y}_{72}$ : Bena Tshibwabwa, $\mathrm{Y}_{73}$ : Bwena Muntu, $\mathrm{Y}_{74}$ : Bukasa Nkumbi Nkumbi, $\mathrm{Y}_{75}$ : de la Poste, $\mathrm{Y}_{76}$ : Kajiba, $\mathrm{Y}_{77}$ : Kansele, $\mathrm{Y}_{78}$ : Lunga, $\mathrm{Y}_{79}$ : Lusambo, $\mathrm{Y}_{80}:$ M'Tshia, $\mathrm{Y}_{81}$ : Muluma Musulu, $\mathrm{Y}_{82}$ : Ngomba Ngole, Y83: Nkonga, Y84: Nzangula Menji, Y85: OAU / AU, Y86: Siona, Y87 : Tender, Y88: Y89 and Tshidibi Tshikunza: Tshiminyi in Muya township.

The variables under study are:

X01: school located along the main thoroughfare, X02: non erosive school site, X03: erosive school site, X04: 
school without toilet, X05: School with toilet X06: school with garbage management system, X07: school with gutter X08: school without trenches X09: school with shaft X10: school with tap, X11: school without water nor electricity X12: initially residential use building, X13: initially for hotel purposes, X14: initially for school purposes X15: non Government funded school, X16: Catholic agreed school, X17: Protestant agreed school X18: secular accredited private school, X19: Catholic private school and X20: Miba private school.

\section{Data Analysis}

The approach relied on for the analysis of the collected data is the factorial analysis of correspondences and the main components analysis. For the description of factorial axes related data, we are going to rely on the factorial methods. the interpretation will be carried out in keeping with the factors. The total sum of values proper amounts to 0,6304 , in the table $n^{\circ} 2$ below.

Table 2. Les valeurs propres

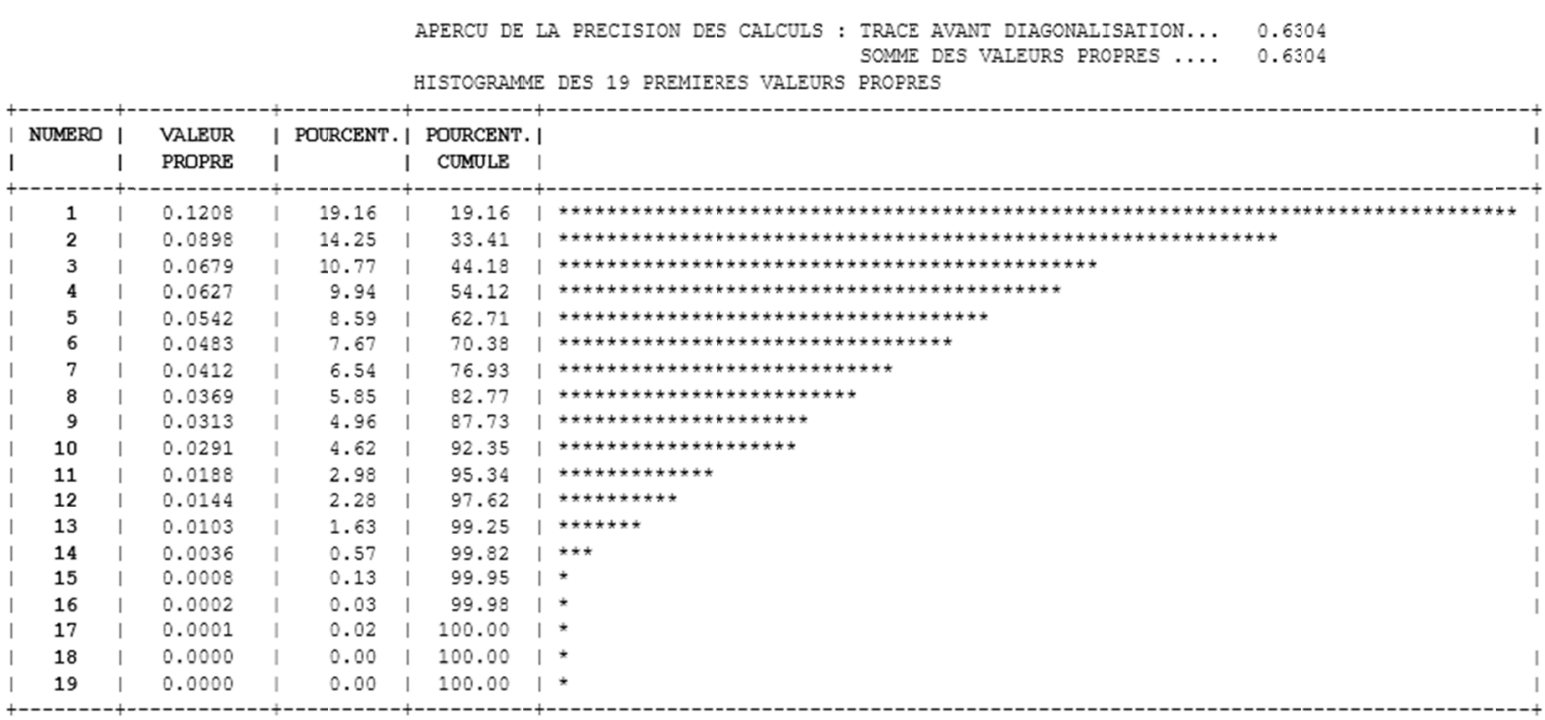

Source: Sorties SPAD

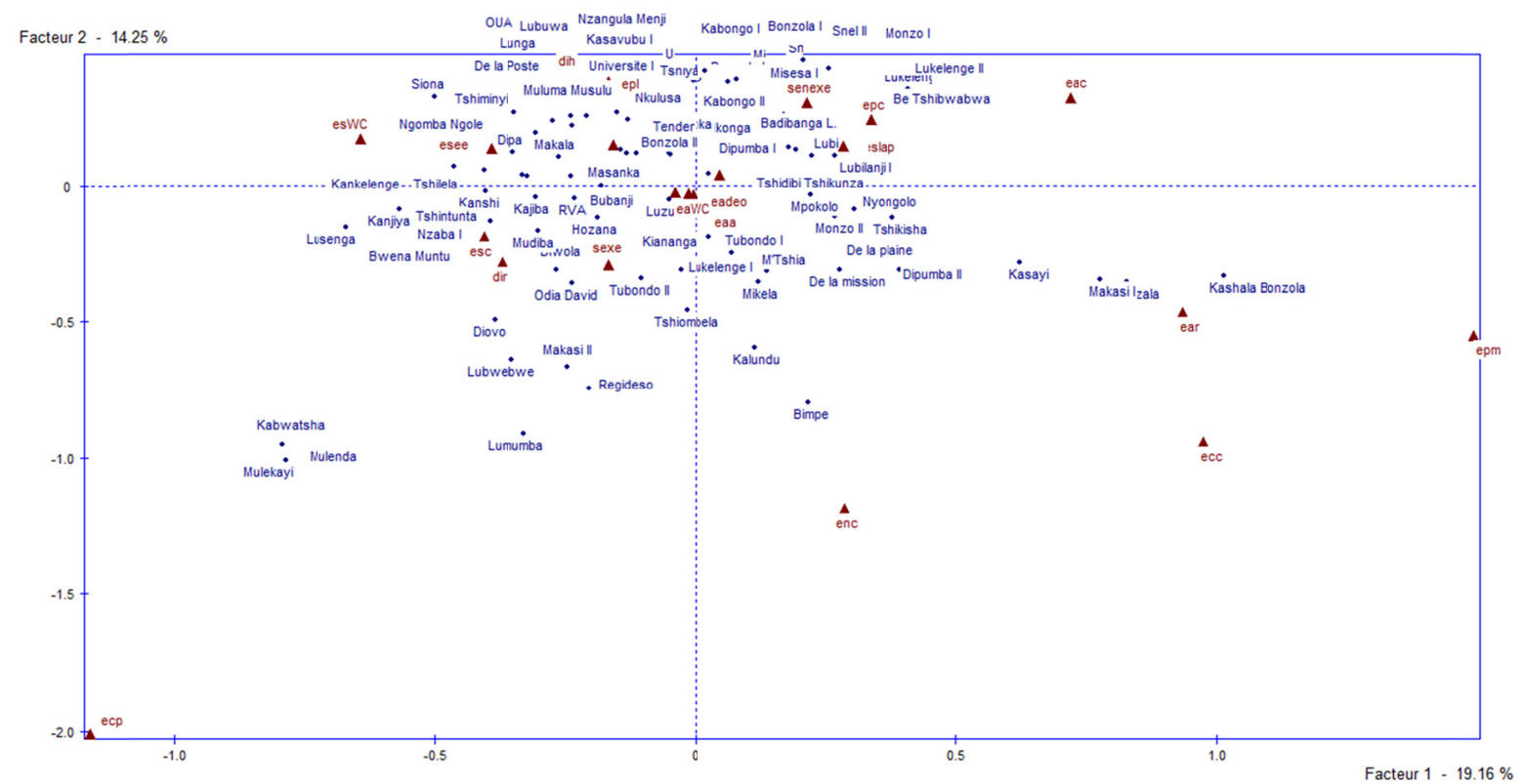

SPAD/Figure 2. Presentation of biplot $\left(1^{\text {st }}\right.$ two axes $=$ Factors $\left.1 \& 2\right)$ 


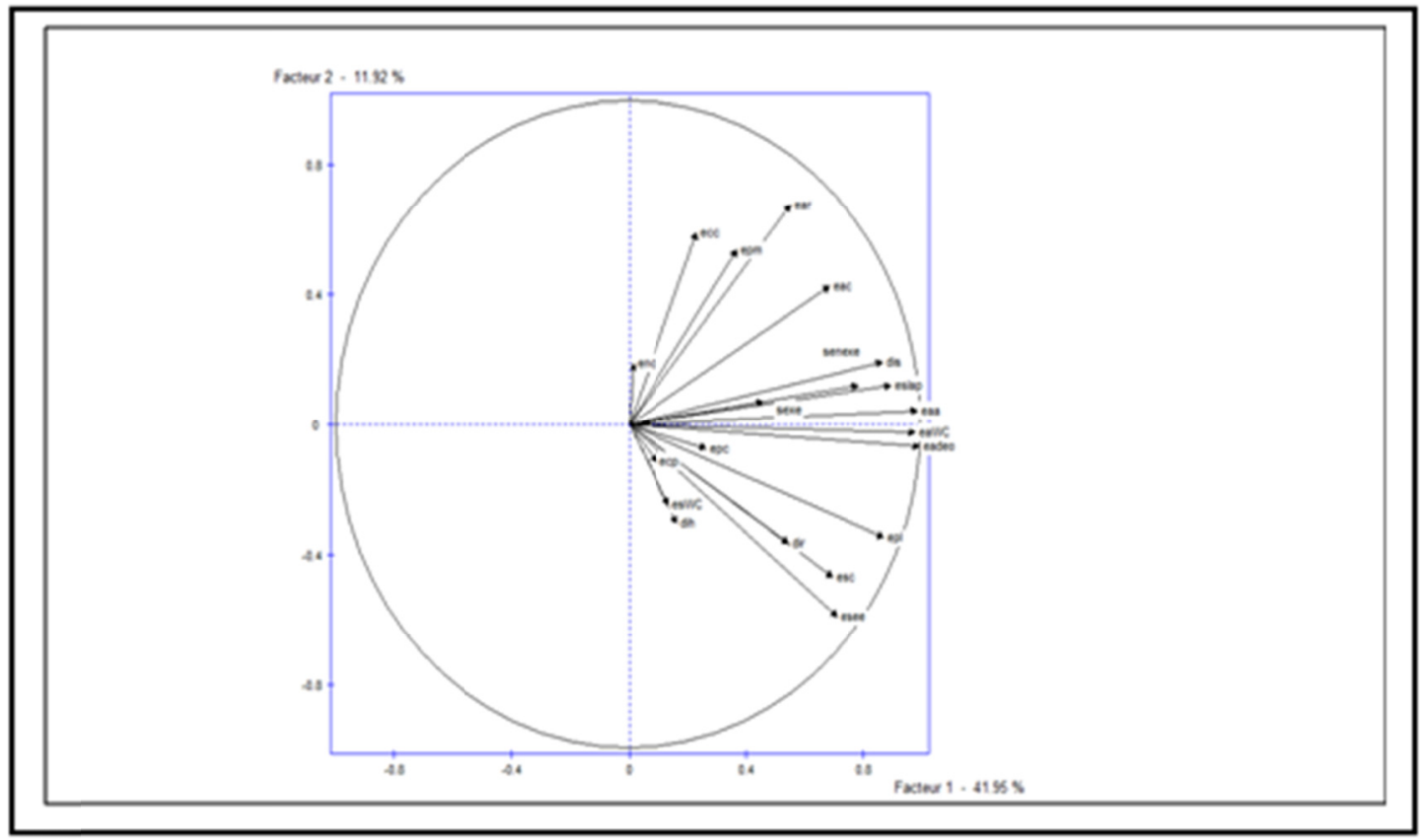

Figure 3. Circle of correlations

Source: SPAD

The factorial design in Figure 3 and the correlation circle in Figure 4 above enable us to see the total inertia of the cloud, with 0.6304 as the total sum of values proper. According to Table 2 above, the first factorial axis represents $19.16 \%$ of the observations and the second axis $14.25 \%$.

In considering the first two axes in Figure 3 which represent, according to Table 2, 33.41\% of information, we can state the following:

- The variable X10 (school with faucet) has contributed to the creation of the first axis;

- The variable X17 (Protestant school) has contributed to the creation of the second axis.

In line with places (Figure 2, Table 1 and Figure 3):

- The quarter Y59 (Kashala Bonzola) in Kanshi commune has amply contributed to the creation of the first axis and

- quarters Y16 (Mulekelayi) and Y17 (Mulenda) in Bipemba commune has contributed more to the creation of the second axis.

In line with the first axis (with $19.16 \%$ of information) Figures 2, 3 and chart 1 show that quarters whose nomenclature has been described on page 6 and in table 1 in the appendix such as Y01, Y02, Y07 and Y15 in Bipemba commune; Y27, Y29, Y30, Y31, Y32, Y35, Y36, Y37, Y39, Y41, Y42, Y44 in Dibindi commune; Y56, Y57, Y58, Y59, Y61, Y62, Y64, Y67, Y68, Y69 in Kanshi commune and Y71, Y72, Y80, Y83, Y88 in Muya commune are characterized by the following variables(see page6): X01, X02, X06, XO7, X09, X10, X15, X16, X19 and X 20.

- The considerable degree of affinity can be explained by the fact that many of these quarters (71\%) belong to two communes, Dibindi and Kanshi. These enjoy the urban amenities. They are no erosive sites along the main arteries with non-subsidized schools, Catholic agreed, Catholic private and Miba private with gutters, trees, garbage management system and tap. In short, they are safe schools, of relatively good quality.

In contrast, quarters Y04, Y05, Y06, Y08, Y09, Y10, Y13, Y14, Y16, Y17, Y19, Y21, Y22, Y23, Y24, Y 25 and Y26 in Bipemba commune; Y28, Y33, Y35, Y45, Y46 and Y47 in the Dibindi commune; Y48, Y49, Y50, Y51, Y52, Y53, Y54 and Y55 in Diulu commune; Y60, Y63, Y64 and Y66 in Kanshi commune and Y73, Y75, Y76, Y78, Y81, Y82, Y84, Y85, Y86, Y87, Y89 in Muya commune associate with variables X03, X04, X05, X08, $\mathrm{X} 11, \mathrm{X} 12, \mathrm{X} 13$ and $\mathrm{X} 18$. These are quarters which represent $64 \%$ are part of two communes, Bipemba and 
Muya. They are mostly urban-rural suburbs with erosive school sites, schools in secular management system with or without toilet, without trenches, without water or electricity, housed in historic hotels and / or residential houses. All things considered, these quarters of the city are unclean and precarious to the extreme and contain mediocre quality schools.

Table 1. Geographic information matrix

\begin{tabular}{|c|c|c|c|c|c|c|c|c|c|c|c|c|c|c|c|c|c|c|c|c|c|c|}
\hline \multicolumn{2}{|c|}{ Quartier } & $x_{01}$ & $x_{02}$ & $x_{03}$ & $x_{04}$ & $x_{05}$ & $x_{06}$ & $x_{07}$ & $x_{0 B}$ & $x_{09}$ & $x_{10}$ & $x_{11}$ & $x_{12}$ & $x_{13}$ & $x_{14}$ & $\mathrm{X}_{15}$ & $x_{16}$ & $x_{17}$ & $\mathrm{X}_{18}$ & $x_{19}$ & $x_{20}$ & TOT \\
\hline$Y_{01}$ & $\begin{array}{l}\text { De la } \\
\text { mission }\end{array}$ & 2,000 & 0,000 & 1,000 & 0,000 & 5,000 & 5,000 & 2,000 & 3,000 & 5,000 & 1,000 & 4,000 & 0,000 & 0,000 & 5,000 & 0,000 & 4,000 & 0,000 & 1,000 & 0,000 & 0,000 & 38,000 \\
\hline$Y_{02}$ & De la plaine & 3,000 & 0,000 & 1,000 & 0,000 & 4,000 & 4,000 & 2,000 & 2,000 & 4,000 & 1,000 & 3,000 & 0,000 & 0,000 & 4,000 & 0,000 & 3,000 & 0,000 & 1,000 & 0,000 & 0,000 & 32,000 \\
\hline$Y_{03}$ & \begin{tabular}{|l|} 
Ditunga \\
dietu
\end{tabular} & 0,000 & 1,000 & 0,000 & 0,000 & 3,000 & 3,000 & 0,000 & 3,000 & 3,000 & 0,000 & 3,000 & 2,000 & 0,000 & 1,000 & 0,000 & 0,000 & 0,000 & 3,000 & 0,000 & 0,000 & 22,000 \\
\hline$Y_{04}$ & Diovo & 1,000 & 0,000 & 1.000 & 0.000 & 3.000 & 3,000 & 0.000 & 3.000 & 3,000 & 0,000 & 3.000 & 1,000 & 0.000 & 2,000 & 0,000 & 1,000 & 1.000 & 1,000 & 0,000 & 0,000 & 23,000 \\
\hline$Y_{05}$ & Diwola & 0.000 & 0,000 & 1,000 & 0,000 & 2,000 & 2,000 & 0,000 & 2,000 & 2,000 & 0,000 & 2,000 & 1,000 & 0.000 & 1,000 & 0,000 & 1,000 & 0,000 & 1,000 & 0,000 & 0,000 & 15,000 \\
\hline$Y_{06}$ & Kabwatshia & 0,000 & 1,000 & 0,000 & 0,000 & 2,000 & 2,000 & 0,000 & 2,000 & 2,000 & 0,000 & 2,000 & 1,000 & 0,000 & 1,000 & 0,000 & 0,000 & 2,000 & 0,000 & 0,000 & 0,000 & $\begin{array}{l}15,000 \\
\end{array}$ \\
\hline$Y_{0 z}$ & Kalundu & 1,000 & 1,000 & 1,000 & 0,000 & 4,000 & 4,000 & 1,000 & 3,000 & 4,000 & 1,000 & 3,000 & 2,000 & 0,000 & 1,000 & 2,000 & 2,000 & 0,000 & 0,000 & 0,000 & 0,000 & 30,000 \\
\hline$Y_{\circ 8}$ & Kanjiya & 0,000 & 0,000 & 1,000 & 0,000 & 2,000 & 2,000 & 0,000 & 2,000 & 2,000 & 0,000 & 2,000 & 2,000 & 0,000 & 0,000 & 0,000 & 0,000 & 0,000 & 2,000 & 0,000 & 0,000 & 15,000 \\
\hline$Y_{\circ 9}$ & Kankelenge & 0.000 & 0,000 & 1,000 & 0.000 & 2,000 & 2,000 & 0.000 & 2,000 & 2,000 & 0.000 & 2.000 & 2,000 & 0.000 & 0,000 & 0,000 & 0.000 & 0.000 & 2,000 & 0,000 & 0,000 & 15,000 \\
\hline$Y_{10}$ & Kanshi & 2,000 & 0,000 & 1,000 & 0,000 & 2,000 & 2,000 & 0,000 & 2,000 & 2,000 & 0,000 & 2,000 & 2,000 & 0,000 & 0,000 & 0,000 & 0,000 & 0,000 & 2,000 & 0,000 & 0,000 & $\begin{array}{l}17,000 \\
\end{array}$ \\
\hline$Y_{11}$ & Katu & 0,000 & 0,000 & 1,000 & 0.000 & 2,000 & 2,000 & 0,000 & 2,000 & 2,000 & 0,000 & 2,000 & 0.000 & 0,000 & 2,000 & 0,000 & 0,000 & 0,000 & 2,000 & 0,000 & 0,000 & 15,000 \\
\hline$Y_{12}$ & Lubanda & 0.000 & 0,000 & 1,000 & 0.000 & 2,000 & 2,000 & 0,000 & 2,000 & 2,000 & 0.000 & 2.000 & 0.000 & 0.000 & 2,000 & 0.000 & 0,000 & 0.000 & 2,000 & 0,000 & 0,000 & $\begin{array}{l}15,000 \\
\end{array}$ \\
\hline$Y_{13}$ & Lubwebwe & 0.000 & 1,000 & 1,000 & 0.000 & 2.000 & 2,000 & 0.000 & 2.000 & 2,000 & 0,000 & 2,000 & 0.000 & 1,000 & 1,000 & 0,000 & 1.000 & 1,000 & 0.000 & 0,000 & 0,000 & 16,000 \\
\hline$Y_{14}$ & Makala & 2,000 & 0,000 & 1,000 & 0.000 & 2,000 & 2.000 & 0,000 & 2,000 & 2,000 & 0.000 & 2,000 & 0.000 & 0.000 & 2,000 & 0,000 & 0.000 & 0.000 & 2,000 & 0,000 & 0,000 & 17,000 \\
\hline$Y_{15}$ & Mpokolo & 3,000 & 1,000 & 1,000 & 0,000 & 4,000 & 4,000 & 2,000 & 2,000 & 4,000 & 0,000 & 4,000 & 0,000 & 0,000 & 4,000 & 0,000 & 2,000 & 0,000 & 0,000 & 2,000 & 0,000 & $\begin{array}{l}33,000 \\
\end{array}$ \\
\hline$Y_{16}$ & Mulekayi & 0,000 & 0,000 & 1,000 & 0,000 & 2,000 & 2,000 & 0,000 & 2,000 & 2,000 & 0,000 & 2,000 & 0,000 & 0,000 & 2,000 & 0,000 & 0,000 & 2,000 & 0,000 & 0,000 & 0,000 & 15,000 \\
\hline$Y_{17}$ & Mulenda & 0,000 & 0,000 & 1,000 & 0,000 & 2,000 & 2,000 & 0,000 & 2,000 & 2,000 & 0,000 & 2,000 & 0,000 & 0,000 & 2,000 & 0,000 & 0,000 & 2,000 & 0,000 & 0,000 & 0,000 & 15,000 \\
\hline$Y_{18}$ & Muya & 0,000 & 0,000 & 1,000 & 0,000 & 2,000 & 2,000 & 0,000 & 2,000 & 2,000 & 0,000 & 2,000 & 0,000 & 0,000 & 2,000 & 0,000 & 0,000 & 0,000 & 2,000 & 0,000 & 0,000 & 15,000 \\
\hline$Y_{19}$ & \begin{tabular}{|l|} 
Nzaba I \\
\end{tabular} & 0,000 & 1,000 & 1,000 & 0.000 & 2.000 & 2,000 & 0.000 & 2,000 & 2,000 & 0,000 & 2.000 & 0.000 & 0.000 & 2,000 & 0,000 & 0,000 & 0.000 & 1,000 & 0,000 & 0,000 & $\begin{array}{l}15,000 \\
\end{array}$ \\
\hline$Y_{20}$ & Nzaba II & 0.000 & 0,000 & 1,000 & 0.000 & 2,000 & 2,000 & 0,000 & 2,000 & 2,000 & 0,000 & 2,000 & 0.000 & 0,000 & 2,000 & 0,000 & 0,000 & 0.000 & 0,000 & 0,000 & 0,000 & 13,000 \\
\hline$\gamma_{21}$ & Odia David & 1,000 & 0,000 & 1,000 & 0,000 & 3,000 & 3,000 & 0,000 & 3,000 & 3,000 & 0,000 & 3,000 & 0,000 & 0,000 & 3,000 & 2,000 & 0,000 & 0,000 & 1,000 & 0,000 & 0,000 & 23,000 \\
\hline$Y_{22}$ & Regideso & 1,000 & 0,000 & 1,000 & 0,000 & 2,000 & 2,000 & 0,000 & 2,000 & 2,000 & 1,000 & 1,000 & 0,000 & 0,000 & 2,000 & 1,000 & 0,000 & 1,000 & 1,000 & 0,000 & 0,000 & 17,000 \\
\hline$Y_{23}$ & RVA & 2,000 & 0,000 & 1,000 & 0,000 & 2,000 & 2,000 & 0,000 & 2,000 & 2,000 & 0,000 & 2,000 & 0,000 & 0,000 & 2,000 & 0,000 & 0,000 & 0,000 & 0,000 & 0,000 & 0,000 & 15,000 \\
\hline$Y_{24}$ & Tshilela & 0,000 & 0,000 & $c, 000$ & 0,000 & 2,000 & 2,000 & 0,000 & 2,000 & 2,000 & 0,000 & 2,000 & 0,000 & 0,000 & 2,000 & 0,000 & 0,000 & 0,000 & 0,000 & 0,000 & 0,000 & 12,000 \\
\hline$Y_{25}$ & Tshintunta & 0,000 & 0,000 & 0,000 & 0.000 & 2,000 & 2,000 & 0,000 & 2,000 & 2,000 & 0.000 & 2,000 & 0,000 & 0,000 & 2,000 & 0,000 & 0,000 & 0.000 & 0,000 & 0,000 & 0,000 & 12,000 \\
\hline$Y_{26}$ & Tshiombela & 1,000 & 0,000 & 1,000 & 0.000 & 2,000 & 2,000 & 0,000 & 2,000 & 2,000 & 1,000 & 1,000 & 0,000 & 1,000 & 1,000 & 1,000 & 0,000 & 0.000 & 0,000 & 0,000 & 0,000 & 15,000 \\
\hline$Y_{22}$ & Bonzola I & 1,000 & 1,000 & 1,000 & 0,000 & 2,000 & 2,000 & 2,000 & 0,000 & 2,000 & 0,000 & 2,000 & 0,000 & 1,000 & 1,000 & 0,000 & 0,000 & 0,000 & 2,000 & 0,000 & 0,000 & 16,000 \\
\hline$Y_{28}$ & Bonzola II & 1,000 & 0,000 & 1,000 & 0,000 & 1,000 & 1,000 & 1,000 & 0,000 & 2,000 & 0,000 & 1,000 & 1,000 & 0,000 & 0,000 & 0,000 & 0,000 & 0,000 & 1,000 & 0,000 & 0,000 & 9,000 \\
\hline$Y_{29}$ & Dipumba I & 4,000 & 3,000 & 1,000 & 0.000 & 4.000 & 4,000 & 2,000 & 2,000 & 4,000 & 2,000 & 2,000 & 1,000 & 0,000 & 3,000 & 0,000 & 0,000 & 0.000 & 3,000 & 0,000 & 0,000 & $\begin{array}{l}35,000 \\
\end{array}$ \\
\hline$r_{30}$ & \begin{tabular}{|l|l} 
Dipumba II \\
\end{tabular} & 2,000 & 1,000 & 1,000 & 0,000 & 2,000 & 2,000 & 0,000 & 2,000 & 2,000 & 2,000 & 0,000 & 0,000 & 0,000 & 2,000 & 0,000 & 1,000 & 0,000 & 1,000 & 0,000 & 0,000 & 18,000 \\
\hline$Y_{31}$ & Kabongol & 3,000 & 3,000 & 0,000 & 1,000 & 2,000 & 3.000 & 2,000 & 1,000 & 3,000 & 0.000 & 3,000 & 0.000 & 0.000 & 3,000 & 0,000 & 0.000 & 0.000 & 3.000 & 0,000 & 0,000 & 27,000 \\
\hline$r_{32}$ & Kabongo II & 5,000 & 5,000 & 0,000 & 0.000 & 5,000 & 5,000 & 3.000 & 2,000 & 5,000 & 0,000 & 5,000 & 0.000 & 0.000 & 5,000 & 0,000 & 0,000 & 0,000 & 5,000 & 0,000 & 0,000 & 45,000 \\
\hline$Y_{33}$ & Kasavubu I & 4,000 & 6,000 & 3,000 & 0.000 & 9,000 & 9,000 & 3,000 & 6,000 & 9,000 & 0,000 & 9,000 & 0.000 & 0.000 & 9,000 & 0,000 & 0,000 & 0.000 & 9,000 & 0,000 & 0,000 & 76,000 \\
\hline$Y_{34}$ & Kasavubu II & 0,000 & 0,000 & 1,000 & 0,000 & 1,000 & 1,000 & 0,000 & 1,000 & 1,000 & 0,000 & 1,000 & 1,000 & 0,000 & 0,000 & 0,000 & 0,000 & 0,000 & 1,000 & 0,000 & 0,000 & 8,000 \\
\hline$Y_{35}$ & Lukelenge I & 2,000 & 2,000 & 0,000 & 0,000 & 2,000 & 2,000 & 0,000 & 2,000 & 2,000 & 0,000 & 2,000 & 0,000 & 1,000 & 1,000 & 1,000 & 1,000 & 0,000 & 0,000 & 0,000 & 0,000 & 18,000 \\
\hline$Y_{36}$ & Lukelenge II & 4.000 & 4,000 & 0,000 & 1,000 & 3.000 & 4.000 & 4,000 & 0.000 & 4,000 & 2,000 & 2,000 & 0.000 & 0,000 & 4,000 & 0,000 & 0,000 & 0.000 & 3,000 & 1,000 & 0,000 & 36,000 \\
\hline$\gamma_{37}$ & Mikela. & 4,000 & 5,000 & 0,000 & 1,000 & 4,000 & 5,000 & 1,000 & 4,000 & 5,000 & 4,000 & 1,000 & 1,000 & 0,000 & 4,000 & 0,000 & 1,000 & 2,000 & 2,000 & 0,000 & 0,000 & 44,000 \\
\hline$Y_{38}$ & Minkoka & 5,000 & 4,000 & 3,000 & 0.000 & 9.000 & 9,000 & 4,000 & 5,000 & 9,000 & 2,000 & 7,000 & 1,000 & 0,000 & 8,000 & 0,000 & 0,000 & 0,000 & 9,000 & 0,000 & 0,000 & 75,000 \\
\hline$Y_{39}$ & Misesa I & 3,000 & 5,000 & 0,000 & 0,000 & 5,000 & 5,000 & 5,000 & 0,000 & 5,000 & 0,000 & 5,000 & 1,000 & 0,000 & 4,000 & 0,000 & 0,000 & 0,000 & 5,000 & 0,000 & 0,000 & $\begin{array}{l}43,000 \\
\end{array}$ \\
\hline $\mathbf{Y}_{40}$ & Misesa II & 4,000 & 4,000 & 3,000 & 1,000 & 6,000 & 7,000 & 2,000 & 5,000 & 7,000 & 0,000 & 7,000 & 1,000 & 0,000 & 6,000 & 0,000 & 1,000 & 0,000 & 6,000 & 0,000 & 0,000 & 60,000 \\
\hline$Y_{41}$ & Monzo I & 1.000 & 2,000 & 0,000 & 0.000 & 2,000 & 2,000 & 2,000 & 0.000 & 2,000 & 0.000 & 2.000 & 0.000 & 0.000 & 2,000 & 0,000 & 0.000 & 0.000 & 1.000 & 1,000 & 0,000 & $\begin{array}{l}17,000 \\
\end{array}$ \\
\hline$Y_{42}$ & Monzo II & 4,000 & 1,000 & 4,000 & 0,000 & 5,000 & 5,000 & 1,000 & 4,000 & 5,000 & 5,000 & 0,000 & 0,000 & 0,000 & 5,000 & 0,000 & 0,000 & 0,000 & 4,000 & 1,000 & 0,000 & 44,000 \\
\hline$Y_{43}$ & Snel I & 8,000 & 6,000 & 5,000 & 0,000 & 11,000 & 11,000 & 10,000 & 1,000 & $\begin{array}{l}1,000 \\
\end{array}$ & 3,000 & 8,000 & 1,000 & 0,000 & 10,000 & 0,000 & 1,000 & 0,000 & 10,000 & 0,000 & 0,000 & 96,000 \\
\hline$Y_{44}$ & Snel II & 2,000 & 2,000 & 0,000 & 0,000 & 2,000 & 2,000 & 2,000 & \begin{tabular}{|l|l|} 
\\
\end{tabular} & 2,000 & 0,000 & 2,000 & 0,000 & 0,000 & 2,000 & 0,000 & 0,000 & \begin{tabular}{|l|l|} 
\\
\end{tabular} & 2,000 & 0,000 & 0,000 & 18,000 \\
\hline$Y_{45}$ & Tshiya & 2,000 & 4,000 & 0,000 & \begin{tabular}{|l|}
1,000 \\
\end{tabular} & 5,000 & 6,000 & 5,000 & \begin{tabular}{|l|}
1,000 \\
\end{tabular} & 6,000 & 0,000 & 6,000 & 1,000 & 0,000 & 5,000 & 0,000 & 0,000 & 0.000 & 6,000 & 0,000 & 0,000 & \begin{tabular}{|l|l}
48,000 \\
\end{tabular} \\
\hline$Y_{46}$ & Univer & 4,000 & 000 & 2,000 & 000 & 000 & 000 & 000 & 000 & 000 & 0,000 & 000 & 0.000 & 0.000 & 000 & 0,000 & 1.000 & 0,000 & 3,000 & 0,000 & 0,000 & 36,000 \\
\hline
\end{tabular}




\begin{tabular}{|c|c|c|c|c|c|c|c|c|c|c|c|c|c|c|c|c|c|c|c|c|c|c|}
\hline$Y_{47}$ & Universite II & 2,000 & 2,000 & 0,000 & 0,000 & 2,000 & 2,000 & 1,000 & 1,000 & 2,000 & 0,000 & 2,000 & 0,000 & 1,000 & 1,000 & 0,000 & 0,000 & 0,000 & 2,000 & 0,000 & 0,000 & 18,000 \\
\hline$Y_{4 a}$ & Bubanji & 4,000 & 1.000 & 0.000 & 0,000 & .000 & 6,000 & 2,000 & 4,000 & 5,000 & 1,000 & 5,000 & 2,000 & 1.000 & 3.000 & 0.000 & 0.000 & 1,000 & 5,000 & 0.000 & 0.000 & 47,000 \\
\hline$Y_{49}$ & Dipa & 4.000 & 0.000 & 0,000 & 0,000 & 000 & 6,000 & 0,000 & 6,000 & 4,000 & 0.000 & 5,000 & 2.000 & 0.000 & 5.000 & 0.000 & 0.000 & 0,000 & 6,000 & 0.000 & 0,000 & 45,000 \\
\hline$Y_{50}$ & Hozana & 1,000 & 1,000 & 0,000 & 0,000 & 2,000 & 2,000 & 0,000 & 2,000 & 1,000 & 0,000 & 2,000 & 0,000 & 0,000 & 2,000 & 1,000 & 0,000 & 0,000 & 1,000 & 0,000 & 0,000 & 15,000 \\
\hline$Y_{51}$ & Lubuwa & 1.000 & 1.000 & 0.000 & 0.000 & 1.000 & 1.000 & 0.000 & 1.000 & 1.000 & 0.000 & 1.000 & 0.000 & 0.000 & 1.000 & 0.000 & 0.000 & 0.000 & 1.000 & 0.000 & 0.000 & 9.000 \\
\hline$Y_{52}$ & Lumumba & 1,000 & 0,000 & 1,000 & 1,000 & 1,000 & 2,000 & 0,000 & 2,000 & 2,000 & 1,000 & 1,000 & 2,000 & 0,000 & 0,000 & 0,000 & 1,000 & 1,000 & 0,000 & 0,000 & 0,000 & 16,000 \\
\hline$Y_{53}$ & Lusenga & 1,000 & 0,000 & 0,000 & 2,000 & 1,000 & 3,000 & 0,000 & 3,000 & 2,000 & 0,000 & $3,00 \mathrm{~b}$ & 0,000 & 0,000 & 3,000 & 0,000 & 0,000 & 1,000 & 2,000 & 0,000 & 0,000 & 21,000 \\
\hline$Y_{54}$ & Masanka & 12,000 & 5,000 & 1,000 & 1,000 & 6,000 & 17,000 & 3,000 & 14,000 & 12,000 & 2,000 & 15,000 & 9,000 & 1,000 & 6,000 & 1,000 & 0,000 & 1,000 & 14,000 & 1,000 & 0,000 & 131,000 \\
\hline$Y_{55}$ & Nkulusa & 3,000 & 4,000 & 1,000 & 0,000 & 6,000 & 6,000 & 1,000 & 5,000 & 5,000 & 0,000 & 6,000 & 1,000 & 0,000 & 5,000 & 0,000 & 1,000 & 0,000 & 4,000 & 1,000 & 0,000 & 49,000 \\
\hline$Y_{56}$ & Bimpe & 0.000 & 1,000 & 0.000 & 0,000 & 1,000 & 1,000 & 0,000 & 1,000 & 1,000 & 1,000 & 0,000 & 1,000 & 0.000 & 0,000 & 1,000 & 0,000 & 0,000 & 0,000 & 0,000 & 0,000 & 8,000 \\
\hline$r_{57}$ & Buzala & 3,000 & 6,000 & 1,000 & 0,000 & 7,000 & 7,000 & 6,000 & 1,000 & 7,000 & 6,000 & 0,000 & 1,000 & 0,000 & 6,000 & 1,000 & 6,000 & 0,000 & 1,000 & 0,000 & 0,000 & 59,000 \\
\hline$Y_{5 s}$ & Kasayi & 1,000 & 3,000 & 0,000 & 0,000 & 3,000 & 3,000 & 2,000 & 1,000 & 3,000 & 3,000 & 0,000 & 1,000 & 0,000 & 2,000 & 1,000 & 1,000 & 0,000 & \begin{tabular}{|l|}
1,000 \\
\end{tabular} & 0,000 & 0,000 & 25,000 \\
\hline $\mathrm{Y}_{59}$ & $\begin{array}{l}\text { Kashala } \\
\text { Bonzola }\end{array}$ & 7,000 & 3,000 & 0,000 & 0,000 & 7,000 & 7,000 & 7,000 & 0,000 & 7,000 & 7,000 & 0,000 & 0,000 & 0,000 & 7,000 & 1,000 & 5,000 & 0,000 & 0,000 & 0,000 & 1,000 & 59,000 \\
\hline$Y_{50}$ & Kianango & 0,000 & 0,000 & 0,000 & 0,000 & 1,000 & 1,000 & 0,000 & 1,000 & 1,000 & 1,000 & 0,000 & 1,000 & 0,000 & 0,000 & 0,000 & 0,000 & 0,000 & 1,000 & 0,000 & 0,000 & 7,000 \\
\hline$Y_{61}$ & Lubilanji I & 2,000 & 4,000 & 1,000 & 0,000 & 5,000 & 5,000 & 3,000 & 2,000 & 5,000 & 3,000 & 2,000 & 2,000 & 0,000 & 3,000 & 0,000 & 0,000 & 0,000 & 3,000 & 2,000 & 0,000 & 42,000 \\
\hline$Y_{62}$ & Lubilanji II & 0.000 & 0.000 & 0.000 & 0,000 & 2,000 & 1,000 & 1.000 & 1,000 & 2,000 & 1,000 & 1,000 & 1,000 & 0.000 & 1.000 & 0.000 & 0.000 & 0,000 & 2,000 & 0,000 & 0,000 & 13,000 \\
\hline$Y_{63}$ & Luzumu & 0,000 & 2,000 & 1,000 & 0,000 & 3,000 & 3,000 & 0,000 & 3,000 & 3,000 & 2,000 & 1,000 & 1,000 & 0,000 & 2,000 & 0,000 & 0,000 & 0,000 & 3,000 & 0,000 & 0,000 & 24,000 \\
\hline$Y_{64}$ & Makasi I & 1,000 & 1,000 & 0,000 & 0,000 & 1,000 & 1,000 & 1,000 & 0,000 & 1,000 & 1,000 & 0,000 & 0,000 & 0,000 & $1,00 d$ & 1,000 & 0,000 & 0,000 & 0,000 & 0,000 & 0,000 & 9,000 \\
\hline$Y_{65}$ & Makasi II & 0,000 & 0,000 & 1,000 & 0,000 & 1,000 & 1,000 & 0,000 & 1,000 & 1,000 & 0,000 & 1,000 & 0,000 & 0,000 & 1,000 & 1,000 & 0,000 & 0,000 & 0,000 & 0,000 & 0,000 & 8,000 \\
\hline$Y_{56}$ & Mudiba & 0,000 & 0,000 & 0,000 & 0,000 & 2,000 & 2,000 & 0,000 & 2,000 & 2,000 & 1,000 & 1,000 & 2,000 & 0,000 & 0,000 & 0,000 & 0,000 & 0,000 & 2,000 & 0,000 & 0,000 & 14,000 \\
\hline$Y_{67}$ & Nyongolo & 1,000 & 2,000 & 1,000 & 0,000 & 3,000 & 3,000 & 1,000 & 2,000 & 3,000 & 2,000 & 1,000 & 1,000 & 0,000 & 2,000 & 0,000 & 0,000 & 0,000 & 2,000 & 0,000 & 1,000 & 25,000 \\
\hline$Y_{E S}$ & Tshikisha & 11,000 & 9,000 & 2,000 & 0,000 & 11,000 & 11,000 & 3,000 & 8,000 & 11,000 & 11,000 & 0,000 & 3,000 & 0,000 & 8,000 & 0,000 & 0,000 & 1,000 & 8,000 & 0,000 & 2,000 & 99,000 \\
\hline$Y_{69}$ & Tubondo I & 1,000 & 1,000 & 2,000 & 0,000 & 3,000 & 3,000 & 1,000 & 2,000 & 3,000 & 1,000 & 2,000 & 1,000 & 0,000 & 2,000 & 1,000 & 0,000 & 0,000 & 1,000 & 1,000 & 0,000 & 25,000 \\
\hline$Y_{70}$ & Tubondo II & 0,000 & 0,000 & 3,000 & 0,000 & 3,000 & 3,000 & 0,000 & 3,000 & 3,000 & 1,000 & 2,000 & 1,000 & 0,000 & 2,000 & 0,000 & 1,000 & 0,000 & 1,000 & 1,000 & 0,000 & 24,000 \\
\hline$Y_{71}$ & $\begin{array}{l}\text { Badibanga } \\
\text { L. }\end{array}$ & 2,000 & 1,000 & 1,000 & 0,000 & 2,000 & 2,000 & 1,000 & 1,000 & 2,000 & 1,000 & 1,000 & 0,000 & 0.000 & 2,000 & 0,000 & 0,000 & 0,000 & 2,000 & 0,000 & 0,000 & 18,000 \\
\hline$Y_{72}$ & Tshibwabwa & 5,000 & 5,000 & 0,000 & 0,000 & 5,000 & 5,000 & 4,000 & 1,000 & 5,000 & 2,000 & 3,000 & 0,000 & 0,000 & 5,000 & 0,000 & 0,000 & 0,000 & 4,000 & 1,000 & 0,000 & 45,000 \\
\hline$Y_{73}$ & $\begin{array}{l}\text { Bwena } \\
\text { Muntu }\end{array}$ & 2,000 & 2,000 & 1.000 & 2,000 & 1,000 & 3,000 & 0.000 & 3,000 & 3.000 & 0.000 & 3,000 & 0.000 & 0.000 & 3.000 & 1.000 & 0.000 & 0.000 & 2,000 & 0.000 & 0.000 & 26,000 \\
\hline$Y_{74}$ & $\begin{array}{l}\text { Bukasa } \\
\text { Nkumbi2 }\end{array}$ & 1,000 & 2,000 & 0,000 & 0,000 & 2,000 & 2,000 & 0,000 & 2,000 & 2,000 & 0,000 & 2,000 & 0,000 & 0,000 & 2,000 & 0,000 & 0,000 & 0,000 & 2,000 & 0,000 & 0,000 & 17,000 \\
\hline$Y_{75}$ & De la Poste & 2,000 & 4,000 & 0,000 & 0,000 & 4,000 & 4,000 & 1,000 & 3,000 & 4,000 & 0,000 & 4,000 & 3,000 & 1,000 & 0,000 & 0,000 & 0,000 & 0,000 & 4,000 & 0,000 & 0,000 & 34,000 \\
\hline$Y_{76}$ & Kajiba & 0,000 & 2,000 & 0,000 & 1,000 & 1,000 & 2,000 & 0,000 & 2,000 & 2,000 & 0,000 & 2,000 & 0,000 & 0,000 & 2,000 & 1,000 & 0,000 & 0,000 & 1,000 & 0,000 & 0,000 & 16,000 \\
\hline$Y_{77}$ & Kansele & 5,000 & 6,000 & 1,000 & 0,000 & 7,000 & 7,000 & 0,000 & 7,000 & 7,000 & 1,000 & 6,000 & 0,000 & 2,000 & 5,000 & 0,000 & 1,000 & 0,000 & 6,000 & 0,000 & 0,000 & 51,000 \\
\hline$Y_{78}$ & Lunga & 0,000 & 2,000 & 0,000 & 0,000 & 2,000 & 2,000 & 0,000 & 2,000 & 2,000 & 0,000 & 2,000 & 0,000 & 0,000 & 2,000 & 0,000 & 0,000 & 0,000 & 2,000 & 0,000 & 0,000 & 16,000 \\
\hline$Y_{70}$ & lusambn & 1,000 & 2,000 & 0,000 & 0,000 & 2,000 & 2,000 & 1,000 & 1,000 & 2,000 & 1,000 & 1,000 & 0,000 & 1,000 & 1,000 & 0,000 & 0,000 & 0,000 & 2,000 & 0,000 & 0,000 & 17,000 \\
\hline$\gamma_{g a}$ & M'Tshia & 0.000 & 1,000 & 0.000 & 0,000 & 1,000 & 1,000 & 0,000 & 1,000 & 1.000 & 0,000 & 1,000 & 0.000 & 0.000 & 1,000 & 0.000 & 1.000 & 0,000 & 0,000 & 0,000 & 0.000 & 8,000 \\
\hline$Y_{81}$ & $\begin{array}{l}\text { Muluma } \\
\text { Musulu }\end{array}$ & 1,000 & 4,000 & 1,000 & 1,000 & 4,000 & 5,000 & 1,000 & 4,000 & 5,000 & 0,000 & 5,000 & 0,000 & 0,000 & 5,000 & 0,000 & 0,000 & 0,000 & 5,000 & 0,000 & 0,000 & 41,000 \\
\hline$Y_{\theta 2}$ & $\begin{array}{l}\text { Ngomba } \\
\text { Ngole }\end{array}$ & 0.000 & 6.000 & 0.000 & 0,000 & 6,000 & 6,000 & 0,000 & 6,000 & 5,000 & 0.000 & 6,000 & 2,000 & 1.000 & 3.000 & 0.000 & 0.000 & 0.000 & 5,000 & 1,000 & 0,000 & 48,000 \\
\hline$Y_{83}$ & Nkonga & 2,000 & 1,000 & 2,000 & 0,000 & 3,000 & 3,000 & 2,000 & 1,000 & 3,000 & 2,000 & 1,000 & 0,000 & 2,000 & 1,000 & 0,000 & 0,000 & 0,000 & 3,000 & 0,000 & 0,000 & 26,000 \\
\hline$Y_{24}$ & $\begin{array}{l}\text { Nzangula } \\
\text { Menji }\end{array}$ & 1,000 & 1,000 & 0,000 & 0,000 & 1,000 & 1,000 & 0,000 & 1,000 & 1,000 & 0,000 & 1,000 & 0,000 & 0.000 & 1,000 & 0,000 & 0,000 & 0,000 & 1,000 & 0,000 & 0,000 & 9,000 \\
\hline$\gamma_{\mathrm{BS}}$ & OUA & 0.000 & 1,000 & 0,000 & 0,000 & 1,000 & 1,000 & 0,000 & 1,000 & 1,000 & 0,000 & 1,000 & 0,000 & 0,000 & 1,000 & 0,000 & 0,000 & 0,000 & 1,000 & 0,000 & 0,000 & 8,000 \\
\hline$Y_{86}$ & Siona & 0,000 & 1,000 & 0,000 & 1,000 & 0,000 & 1,000 & 0,000 & 1,000 & 1,000 & 0,000 & 1,000 & 0,000 & 0,000 & 1,000 & 0,000 & 0,000 & 0,000 & 1,000 & 0,000 & 0,000 & 8,000 \\
\hline$Y_{87}$ & Tender & 3,000 & 2,000 & 1,000 & 0,000 & 3,000 & 3,000 & 0,000 & 3,000 & 3,000 & 1,000 & 2,000 & 0,000 & 0,000 & 3,000 & 0,000 & 0,000 & 0,000 & 3,000 & 0,000 & 0,000 & 27,000 \\
\hline$Y_{B s}$ & $\begin{array}{l}\text { Tshidibi } \\
\text { Tshikunza }\end{array}$ & 0,000 & 1,000 & 0,000 & 0,000 & 1,000 & 1,000 & 0,000 & 1,000 & 1,000 & 1,000 & 0,000 & 0,000 & 0,000 & 1,000 & 0,000 & 0,000 & 0,000 & 1,000 & 0,000 & 0,000 & 8,000 \\
\hline$Y_{89}$ & Tshiminyi & 0,000 & 3,000 & 0,000 & 1,000 & 2,000 & 3.000 & 0,000 & 3,000 & 3,000 & 0,000 & 3,000 & 0,000 & 0.000 & 3.000 & 0.000 & 0.000 & 0,000 & 3,000 & 0,000 & 0,000 & 24,000 \\
\hline To & onne & 3.000 & 9,000 & 69,000 & 17,000 & 288,000 & 304,000 & 99,000 & 206,000 & 294,000 & 79,000 & 225,000 & 59,000 & 14,000 & 231,000 & 18,000 & 37,000 & 16,000 & 208,000 & 13,000 & 4,000 & 2503 \\
\hline
\end{tabular}

Source: - Inquiry on plot, 2008, 2009, 2013 et 2014

- For the nomenclature of variables see pages $6 \& 7$ 
As far as the second axis is concerned (with 14.25\% of information in Table 2), Figure 1, Table 1 and Figure 2 show that 1st quadrant quarters (Y27, Y29, Y31, Y32, Y35, Y36, Y39, Y41 and Y44 in Dibindi commune, Y61 and Y62 in Kanshi commune and Y71, Y72, Y83 and Y88 in Muya commune associated with variables X01, X02, X07, X19 are, mostly quarters containing sanitized schools. Moreover, the second quadrant quarters (Y09, Y14 and Y24 in Bipemba commune, Y28, Y33, Y45, Y46 and Y47 in Dibindi commune, Y49, Y51, Y54 and Y55 in Diulu commune and Y75, Y78, Y81, Y82, Y84, Y85, Y86, Y87 and Y89 in Muya commune) associated with variables X04, X11, X13 and X18 contain unclean schools, which is indicative of poor environment.

By contrast, the 3rd quadrant quarters (Y04, Y05, Y06, Y08, Y10, Y13, Y16, Y17, Y19, Y21, Y22, Y23, Y25 and Y26 in Bipemba commune, Y35 in Dibindi commune, Y48, Y50, Y52, Y53 in Diulu commune, Y60, Y63, Y65, Y66 and Y70 in Kanshi commune, Y73 and Y76 in Muya commune) combined with variables X03, X05, X08, X12 and X17 surely contain Protestant agreed substandard schools, in old residential houses, without gutters and on sites exposed to gully erosion but with latrines, though traditional. As for the quarters of the 4th quadrant, Y01, Y02, Y07 and Y15 in Bipemba commune, Y30, Y37 and Y42 in Dibindi commune, Y56, Y57, Y58, Y59, Y64, Y67, Y68 and Y69 in Kanshi commune and Y80 in Muya commune associated with variables X06, X09, $\mathrm{X} 10, \mathrm{X} 15, \mathrm{X} 16$ and X20, they contain non subsidized schools, Catholic agreed and Miba private with trees, with garbage management system and tap, that is, clean and safe schools.

Overall, the interpretation of the first two axes of the factorial design reveals that they represent $33.41 \%$ of information and 1st quadrant quarters associated with variables related thereto (see the analysis of the 1st axis above) are relatively well sanitized quarters containing the renowned schools and also relatively safe. By contrast, quarters of the 3rd quadrant in association with variables relating thereto (see the analysis of the second axis above) are mostly peripheral quarters with substandard schools.

The quarters of the 2nd quadrant, in line with the variable related thereto, (see the analysis of the second axis above) are generally not cleaned quarters of Mbujimayi. They contain essentially secular private schools of the second class. Conversely, quarters of the 4th quadrant variables related thereto (see the analysis of the 1st axis above) are safe quarters containing, clean and fairly well sanitized non-subsidized, Catholic and private-treaty Miba schools

\section{Conclusion}

This research on geographical variation in the quality of school offer in the city of Mbujimayi by multivariate spatial analysis helped to detect the quality of the education system of this city by its infrastructure. Indeed, multivariate analysis of survey data helped to understand that the quality of school offer is determined by a strong interaction between school sanitation and access to public services including running water and electricity. Several maximum associations between variables came to light thanks to solid relationships.

The chains of correlations have shown that there is a spatial disparity between variables with links to the location and topography of adequate school site, sanitation, access to public services and matching of school buildings. Conversely, the variables related to unsanitary, localization and indecent topography of the site and the lack of access to public services have come to the open.

All in all, the study under consideration has allowed us to distinguish two types of quarters in the city of Mbujimayi. On one hand, there are quarters with urban amenities and safe and clean, non-agreed, Catholic agreed, Catholic and private Miba schools on non-erosive sites located along the main thoroughfares. On the other hand, there are peripheral, urban-rural and unsafe quarters containing secular unsafe schools on erosive sites.

It follows from the above that to remedy the precarity of the peripheral quarters and of the schools they contain a balanced, holistic and long lasting sectoral planning of quarters is needed. This includes the programming of investments in road infrastructure equipped with gutters, schools sanitation and their access to public services (running water and electricity. Moreover, maintenance culture should be imparted to managers of public properties, here schools.

\section{References}

Beguin, H. (1979). Méthodes d'analyse géographique quantitative, éd. LITEC, Paris, 252 p. Géographie, éd. Economica, Paris, 497-513

Chadule (Groupe) (1987). Initiation aux méthodes statistiques en géographie, Masson, Paris, $189 \mathrm{p}$.

Escofier, B. \& Pagès, J., (1988). Analyses factorielles simples et multiples. Objectifs, méthodes et interprétation, Dunod, Paris. 
Haring, L. L. et al. (1992). Introduction to scientific geographic research. McGraw Hill, p.18

Kakese, K. et al., (2012). Impact de l'exploitation des géomatériaux sur les populations riveraines dans la ville de Kinshasa: Traitement des données par les méthodes factorielles. Revue Congolaise des Sciences Nucléaires, Volume $26 \mathrm{~N}^{\mathrm{0}}$ 1-Juin 2012

Knox, J. P. \& Marston, S. A., (2001). Places and regions in global context: Human geography. Ed. Printice-Hall, p. 251

Ntumba, B. S. (2010). Contribution au Traitement des données par l'approche Multi-blocs: Analyse du Compromis et Application, Thèse de doctorat en sciences, Université de Kinshasa, 178 p.

Omasombo, T. J. (2014 (dir.)). Le Kasaï Oriental nœud gordien dans l'espace congolais, Musée royal de l'Afrique centrale (MRAC) Tervuren (Belgique), $456 \mathrm{p}$.

Paegelow, M. (1996). Nouvelles technologies en Géographie, Service d'enseignement à distance, Université de Toulouse Le Mirail, Année Universitaire 1995/1996, 181 p.

Pagès J. P. et al. (1979). Analyse factorielle: un peu d'histoire et de géométrie. Revue de Statistique Appliquée, vol. XXVII, $\mathrm{N}^{\mathrm{o}}$, pp. 5-28

Racine, J. B. \& Reymond, H. (1973). L'analyse quantitative en géographie, P.U.F., Collection Sup. "Le géographe". Paris, $316 \mathrm{p}$.

Rubestein, J. M. (1996). The Cultural landscape: An introduction to human geography. $5^{\grave{e}}$ éd. Printice-Hall; p. 493

Tenenhaus, M. (2007). Statistique Méthodes pour décrire, expliquer et prévoir, éd. Dunod, Paris, 679 p.

UNICEF, (2008). Pauvreté des enfants et disparités en République démocratique du Congo. Retrieved from http://www. unicef.org/w caro/w caro R D C Rapport sur la pauvreté des enfants.pdf, consulté le 3 mai 2013

Volle, M. (1978). Analyse des données, Economica, Paris, 267 p.

\section{Copyrights}

Copyright for this article is retained by the author(s), with first publication rights granted to the journal.

This is an open-access article distributed under the terms and conditions of the Creative Commons Attribution license (http://creativecommons.org/licenses/by/4.0/). 